\title{
Ultrastructural Localization of Actin in Nuclear Matrices from Mouse Leukemia L5178Y Cells.
}

\author{
Hiroshi Nakayasu* and Kiyoshi Ueda \\ Department of Medical Biochemistry, Shiga University of Medical Science, \\ Seta Otsu 520-21, Japan
}

\begin{abstract}
We examined the distribution of actin in isolated nuclear matrices from mouse leukemia L5178Y cells using an anti-actin antibody and protein A-conjugated colloidal gold particles. Before immunogold staining, we partially digested the surface lamina of the nuclear matrix with trypsin (Nakayasu and Ueda, Exp. Cell Res. 143, 55-62, 1983) to allow penetration of the gold particles into the nuclear matrix. Trypsin digestion slightly modified the internal structure of the nuclear matrix, but did not affect the actin content in the nuclear matrix nor the reactivity of actin with the antibody. Many colloidal gold particles were present along fibrogranular structures in the nuclear matrix. The results reported here confirm the existence of actin in the interior of the nuclear matrices of L5178Y cells.
\end{abstract}

The isolated nuclear skeletal structure, the nuclear matrix (1), usually contains a considerable amount of actin $(3,9,13)$. Little is known about the function of this nuclear actin, but several recent reports have revealed the involvement of nuclear actin in the synthesis $(6,15)$, processing $(10,12)$ and transport $(13)$ of nascent nuclear RNAs. Therefore, we need to know what the architecture of this actin in the nucleus or nuclear matrix is. The nucleoplasm in the huge nuclei of amphibian oocytes is filled with numerous actin filaments $(4,5)$. Gounon and Karsenti (7) have reported nuclear RNP-like particles associated with these actin filaments in the newt oocyte nucleoplasm.

In contrast, there have been few studies done on the distribution of actin in nuclei of normal size. Recently, our research has suggested that actin is present in a fibrogranular structure, which corresponds to the nucleoplasm, in nuclear matrices of bovine lymphocytes (11). To confirm this, we set out to determine the architecture of the actin in the nuclear matrix by using colloidal gold staining. We found that actin is associated with the fibrogranular structure present in nuclear matrices of mouse leukemia L5178Y cells.

\section{MATERIALS AND METHODS}

Protein A-conjugated colloidal gold (about $25 \mathrm{~nm}$ in diameter) was prepared by reducing tetrachloroauric acid with sodium citrate as described by Roth et al. (14). But, because

* Present address: Department of Biological Sciences, State University of New York at Buffalo, Buffalo, N.Y. 14260, U.S.A. 
the gold particles might be excluded from the nuclear matrix because of nuclear lamina, the surface lamina was partially removed by trypsin treatment as described in (11) to permit penetration of both the anti-actin antibody and the colloidal gold into the sphere.

Nuclear matrices were prepared from L5178Y cells as described in (13). Briefly, cells were disrupted by the use of an isotonic buffer containing $1 \%$ Triton $\mathrm{X}-100$. The cytoplasmic fraction was separated from the nuclei by low speed centrifugation. The isolated nuclei were digested with DNase I (the supernatant, DNase extract), after which the chromatin was extracted with a high salt buffer (the supernatant, high salt extract). The resulting pellet (nuclear matrix, $1 \mathrm{mg}$ protein) was suspended in $0.5 \mathrm{ml}$ of Buffer A (10 mM PIPES buffer, pH 6.9, containing $2 \mathrm{mM} \mathrm{MgCl}_{2}$ and $0.1 \mathrm{M} \mathrm{NaCl}$ ) then digested with $5 \mu \mathrm{g}$ of trypsin at $30^{\circ} \mathrm{C}$ for $2 \mathrm{~min}$. The reaction was stopped by the addition of $10 \mu \mathrm{g}$ of trypsin inhibitor. Little actin was released from the nuclear matrix by this digestion. Immunogold staining was carried out as described by Langanger et al. (8) with the following modifications:

Because trypsin-treated nuclear matrices tend to be broken easily by a mechanical force (e.g., centrifugation or pipetting), they first were fixed with glutaraldehyde $(0.32 \%)$ for $5 \mathrm{~min}$, after which the remaining active aldehyde groups were blocked by the addition of sodium borohydride $(0.1 \%, 5 \mathrm{~min})$ to prevent any non-specific binding of the anti-actin antibody. This mixture was centrifuged at $2,000 \mathrm{rpm}$ for $20 \mathrm{~min}$ then washed twice with Buffer B (10 mM PIPES buffer, pH 6.9 , containing $0.1 \%$ ovalbumin, $2 \mathrm{mM} \mathrm{MgCl}_{2}$ and $0.1 \mathrm{M} \mathrm{NaCl}$ ). The trypsin-treated nuclear matrices were treated with $0.7 \mathrm{mg}$ protein $/ \mathrm{ml}$ of anti-actin antibody (obtained from Biomedical Technologies Inc.) for $2 \mathrm{~h}$ with mild shaking then washed twice with Buffer B. Next, the sample was mixed with protein A-conjugated colloidal gold (O.D. $\left.5_{20}=1.0\right)$ for $2 \mathrm{~h}$ then washed twice with Buffer B. The immunogoldstained nuclear matrices obtained were fixed at $0^{\circ} \mathrm{C}$ for $5 \mathrm{~h}$ with $3.2 \%$ glutaraldehyde and $0.2 \%$ tannic acid $(0.1 \mathrm{M}$ sodium phosphate buffer, $\mathrm{pH} 7.2)$, after which they were fixed with $1 \%$ osmium tetroxide at $4^{\circ} \mathrm{C}$ overnight then dehydrated with ethanol and embedded in an Epon 812 mixture (11).

\section{RESULTS AND DISCUSSION}

The intracellular and intranuclear distribution of actin in L5178Y cells is shown in Fig. 1. Western blotting and an enzyme-linked immunosorbent assay showed the presence of actin both in the cytoplasm and in the nucleus. It was clear that the isolated nuclear matrices did contain actin. Among the subnuclear fractions the actin content was remarkably high in the DNase extract (lane 3) and the nuclear matrices (lane 5), the values of the specific contents of actin in both being higher than the value of the cytoplasmic fraction (lane 2). From the intranuclear distribution of protein, we roughly estimated that the nuclear matrix contains about $30 \%$ of the nuclear actin. As described elsewhere $(11,12)$, actin is resistant to trypsin; but, there was the possibility of actin's reactivity with the anti-actin antibody being decreased by some alteration during trypsin digestion. Western blots of trypsin-treated and untreated nuclear matrices revealed, however, that trypsin digestion affected neither the actin content in the nuclear matrix nor actin's reactivity with the antibody (data not shown).

The localization of actin in the nuclear matrix was examined by immunogold staining (Fig. 2). Slight cytoplasmic contamination was present on the surfaces of isolated nuclei (Fig. 2A). The nuclear matrix (Fig. 2B) has three structurally distinct parts in its sphere: the surface lamina, the internal fibrogranular structure and the 


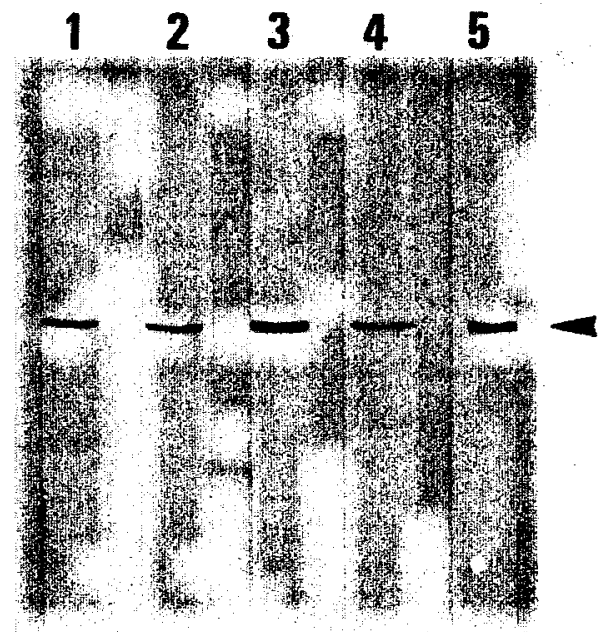

Fig. 1. Detection of actin by Western blotting. Each fraction (lane 2, cytoplasmic fraction; lane 3, DNase extract; lane 4, high salt extract; lane 5, nuclear matrix: $1 \mu \mathrm{g}$ protein each) was prepared as described in the text. Chicken gizzard actin (lane 1, $10 \mathrm{ng}$ protein) was purified by the method of (13). After electrophoresis (9), Western blotting was performed as described previously (13). Actin bands were made visible by an enzyme-linked immunosorbent assay (2) using anti-actin antibody (rabbit) from Biomedical Technologies Inc., and alkaline phosphatase-conjugated anti-rabbit IgG antibody from Miles-Yeda Ltd. The arrowhead indicates the position of actin on the blots.

residual nucleoli. Using weak trypsin digestion, we partially removed the surface lamina (Fig. 2C); neither the fibrogranular structure nor the residual nucleoli was damaged by this digestion (Fig. 2B and 2C). Many gold particles were found in the spheres of the trypsin-treated nuclear matrices (Fig. 2C). This confirms the presence of actin in the interior of the nuclear matrix, as previously suggested $(11,13)$. Control experiments were done with a control serum from another rabbit instead of the anti-actin antibody. In this case, few colloidal gold particles were present in the nuclear matrices (data not shown).

A photograph taken at high magnification (Fig. 2D) shows the distribution of actin in more detail. Many gold particles are located along the fibrogranular structure, which suggests that actin is a constituent of this structure. This agreed with our previous findings (13) which indicated that the fibrogranular structure is altered remarkably by depolymerization of the nuclear matrix actin. Scarcely any colloidal gold particles were detected in the residual nucleoli of the nuclear matrix, which indicate the absence of actin in the nucleoli. But, it is possible that the colloidal gold could not penetrate into the nucleoli because of the high density of these residual nucleoli. Therefore, our results do not necessarily rule out the presence of actin in the nucleoli.

There was the possibility that the nuclear matrix actin might be contaminated with cytoplasmic actin. But, as shown in Fig. 1, the specific actin content in the nuclear matrix fraction was higher than that in the cytoplasmic fraction. Even if cytoplasmic actin had entered the nucleus and been non-specifically adsorbed on its chromatin during nuclear isolation, it should have been removed from the nuclear matrix by 


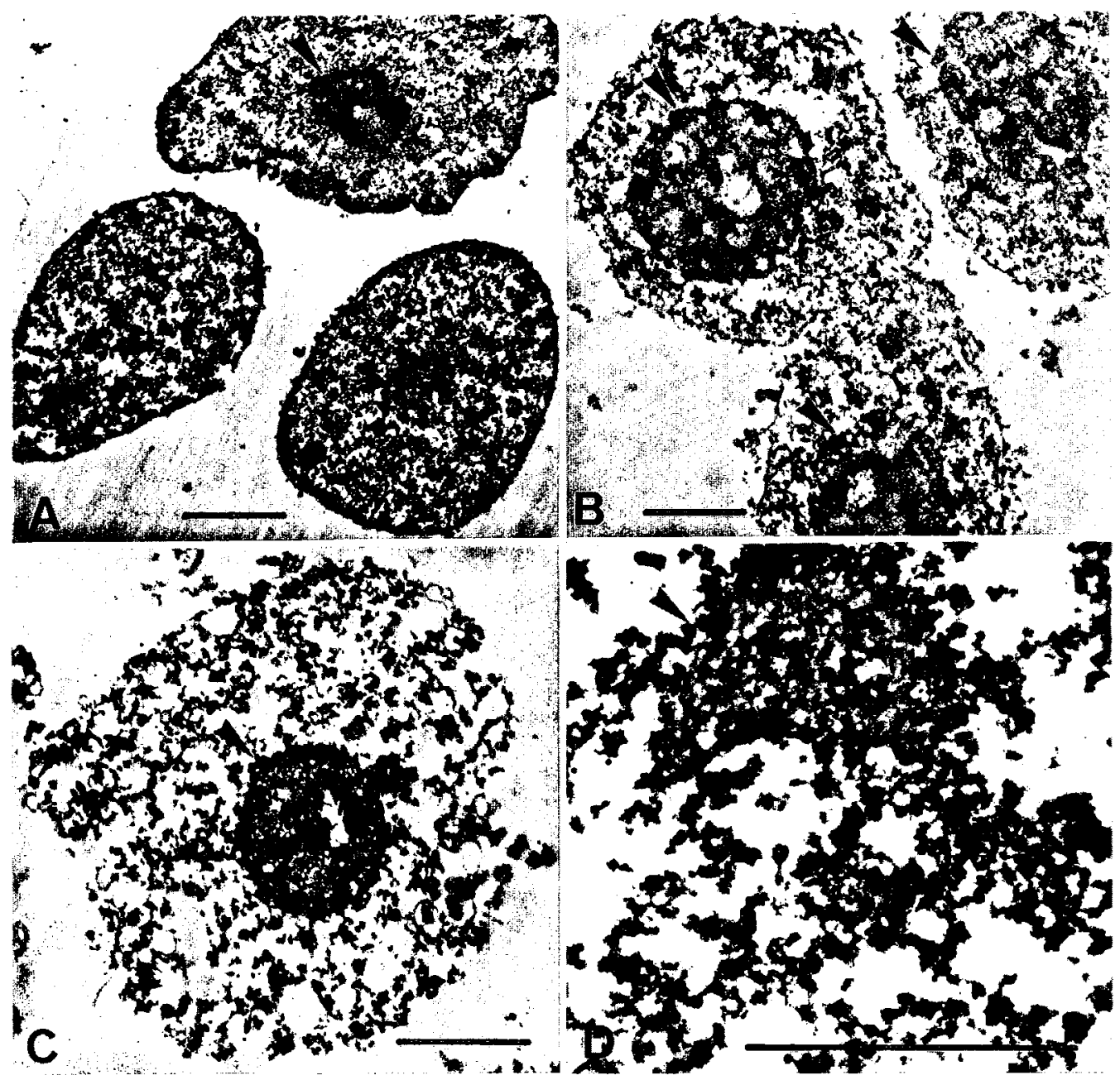

Fig. 2. Electron microscopy of isolated nuclei (A), nuclear matrices (B) and trypsin-treated nuclear matrices $(C, D)$. Samples $C$ and $D$ were fixed after immunogold staining as described in the text; samples A and B were not treated. The arrowheads indicate the nucleoli. Bar, $2 \mu \mathrm{m}$.

the high salt extraction procedure. Therefore, we believe that the labeling of nuclear matrix actin with immunogold particles was not due to contamination by cytoplasmic actin; rather, it shows actin to be a characteristic constituent of the nuclear matrix.

\section{REFERENCES}

1. BEREZNEY, R. and D.S. COFFEY. Nuclear matrix: Isolation and characterization of a framework structure from rat liver nuclei. $J$. Cell Biol. 73, 616-637, 1977

2. Blake, M.S., K.H. Johnston, G.J. Russell-Jones and E.C. Gotschlich. A rapid, sensitive method for detection of alkaline phosphatase-conjugated anti-antibody on Western blots. Anal. Biochem. 136, 175-179, 1984

3. Capco, D.G., K.M. WAN and S. Penman. The nuclear matrix: Three-dimensional architecture and protein composition. Cell 29, 847-858, 1982 
4. Clark, T.G. and R.W. Merriam. Diffusible and bound actin in nuclei of Xenopus laevis oocytes. Cell 12, 883-891, 1977

5. Clark, T.G. and J.L. Rosenbaum. An actin filament matrix in hand-isolated nuclei of Xenopus laevis oocytes. Cell 18, 1101-1108, 1979

6. Egry, J.M., N.G. Miyamoto, V. Moncollin and P. Chambon. Is actin a transcription initiation factor for RNA polymerase B? EMBO J. 3, 2363-2371, 1984

7. GounON, P. and E. KARSENTI. Involvement of contractile proteins in the changes in consistency of oocyte nucleoplasm of the newt Pleurodeles waltiii. J. Cell Biol. 88, 410-421, 1981

8. Langanger, G., J.D. Mey, M. Moeremans, G. Daneels, M.D. Brabander and J.V. Small. Ultrastructural localization of $\alpha$-actinin and filamin in cultured cells with the immunogold staining (IGS) method. J. Cell Biol. 99, 1324-1334, 1984

9. NAKAYASU, H. and K. UEDA. Isolation and characterization of bovine lymphocyte nuclear matrix. Cell Struct. Funct. 6, 181-190, 1981

10. Nakayasu, H., H. Mori and K. Ueda. Association of small nuclear RNA-protein complex with the nuclear matrix from bovine lymphocytes. Cell Struct. Funct. 7, 253-262, 1982

11. Nakayasu, H. and K. Ueda. Association of actin with the nuclear matrix from bovine lymphocytes. Exp. Cell Res. 143, 55-62, 1983

12. Nakayasu, H. and K. Ueda. Small nuclear RNA-protein complex anchors on the actin filaments in bovine lymphocyte nuclear matrix. Cell Struct. Funct. 9, 317-325, 1984

13. NakAyasu, H. and K. Ueda. Association of rapidly-labeled RNAs with actin filaments in nuclear matrix from mouse L5178Y cells. Exp. Cell Res. in press

14. Roth, J., M. Bendayan and L. OrCI. Ultrastructural localization of intracellular antigens by the use of protein A-gold complex. J. Histochem. Cytochem. 26, 1074-1081, 1978

15. Scheer, U., H. HinsSen, W.W. Franke and B.M. JockusCH. Microinjection of actin-binding proteins and actin antibodies demonstrates involvement of nuclear actin in transcription of lampbrush chromosomes. Cell 39, 111-122, 1984

(Received for publication July 16, 1985) 